\title{
Radiation and immunotherapy: a synergistic combination
}

\author{
Anusha Kalbasi, ${ }^{1}$ Carl H. June, ${ }^{2,3}$ Naomi Haas, ${ }^{3}$ and Neha Vapiwala1 \\ 1Department of Radiation Oncology, ${ }^{2}$ Department of Pathology and Laboratory Medicine, and ${ }^{3}$ Division of Hematology/Oncology, \\ Department of Medicine, Hospital of the University of Pennsylvania, Philadelphia, Pennsylvania, USA.
}

\begin{abstract}
Immunotherapy can be an effective treatment for metastatic cancer, but a significant subpopulation will not respond, likely due to the lack of antigenic mutations or the immune-evasive properties of cancer. Likewise, radiation therapy (RT) is an established cancer treatment, but local failures still occur. Clinical observations suggest that RT may expand the therapeutic reach of immunotherapy. We examine the immunobiologic and clinical rationale for combining RT and immunotherapy, two modalities yet to be used in combination in routine practice. Preclinical data indicate that RT can potentiate the systemic efficacy of immunotherapy, while activation of the innate and adaptive immune system can enhance the local efficacy of RT.
\end{abstract}

\section{Introduction}

Over the past two decades, scientific and technological advances in immunotherapy have contributed to its role as one of the more promising cancer treatments. Previously, immunotherapy had been limited to nonspecific agents such as the Bacillus Calmette-Guerin vaccine, IL-2, and interferon- $\gamma$, but the characterization of cancer-specific antigens allowed for the emergence of cancer vaccines and cell-based therapies. In addition, immunobiologists identified checkpoints in immune regulation that led to the development of molecularly targeted approaches in cancer immunotherapy.

Although immunotherapy is enticing because of occasional profound responses, it presently benefits a minority of patients in limited disease sites, and responses can be short lived. New combination approaches are needed to increase the efficacy of immunotherapy and expand its reach to other malignancies. Herein we describe the shortcomings of each immunotherapeutic modality. We then describe how radiation therapy (RT) counters immune evasion and how immunotherapy may potentiate local effects of RT. We also address the logistical aspects of combining RT with immunotherapy and discuss ongoing and future clinical applications.

\section{Immunotherapy: principles and shortcomings}

Mechanisms of tumor immune evasion. Immunotherapy emerged from the basic tenet of tumor immunology that tumors harbor antigens recognized by the immune system (1). This is supported by the observation of concomitant immunity, wherein a host bearing a progressive tumor will reject an inoculum of the same tumor at a distant site $(2,3)$.

The inability of immunotherapy to achieve maximal efficacy is related to immune-evasive properties of tumor cells. Broadly, tumor cells can either decrease their intrinsic immunogenicity or induce tolerance through interactions with the immune system. This tumor-host immune relationship is encompassed by the cancer immunoediting hypothesis, which states that tumors can be both held in check and promoted by the immune system (Figure 1 and refs. 4, 5). Cancer immunoediting can be described by three phases: elimination, equilibrium, and escape. In the elimination phase, transformed cells are quickly recognized and destroyed. We

Conflict of interest: The authors have declared that no conflict of interest exists. Citation for this article: J Clin Invest. 2013;123(7):2756-2763. doi:10.1172/JCI69219. now know that tumors that are not eliminated are "sculpted" by the immune environment in which they develop (4). This tumorhost relationship defines the equilibrium state, until the balance of tumor growth and immune regulation tips and tumor escape prevails. The clinical presence of a tumor suggests a failure in elimination and progression to equilibrium or escape. Immunotherapy aims to shift the tumor from the equilibrium and escape phases to the elimination phase. The examples below illustrate how tumors avoid immunotherapy-mediated elimination.

Vaccine-based strategies. Two studies examined the use of vaccines in metastatic castrate-resistant prostate cancer. In a randomized, phase 3 study from 2006, investigators examined Provenge, a dendritic cell vaccine consisting of peripheral blood mononuclear cells enriched with GM-CSF and prostatic acid phosphatase, a tumor antigen (6). The study randomized 512 patients to the vaccine or a similar product lacking tumor antigen and observed a 4-month benefit in overall survival after 3 years (but no change in progression-free survival). In 2010, a randomized, phase 2 study of poxviral-based prostate cancer vaccine in 112 participants (82 patients, 40 control subjects) demonstrated a median survival increase of 8.5 months and an increase in 3-year overall survival from $18 \%$ to $30 \%$ (7). Most recently, tumor-associated peptide (TUMAP) vaccines have shown a survival benefit in renal cell carcinoma (8).

Cancer vaccine therapy has achieved partial success by modulating a preexisting response to tumor antigens or inducing new antitumor responses. As described above, cancer vaccines appear to affect overall survival more than they mediate objective responses. Although this could be due to the inability of current imaging modalities to distinguish antitumor inflammatory responses from persistent tumors, a more likely explanation is a shift in the immune system from the escape phase to the equilibrium phase, but not to the elimination phase. This failure of elimination can be attributed to the persistence of negative regulatory checkpoints, incomplete amplification of an effector $T$ cell population, or vaccination against an insufficient variety of antigens. Moreover, persistent exposure of antigen without appropriate costimulation can induce peripheral $\mathrm{T}$ cell tolerance and apoptosis (9).

Delivering effector cells. In the 1980s, tumor-infiltrating lymphocytes were isolated, expanded, and used therapeutically in metastatic melanoma (10). Unlike cancer vaccines, which indirectly amplify the effector component of immunity, this approach 


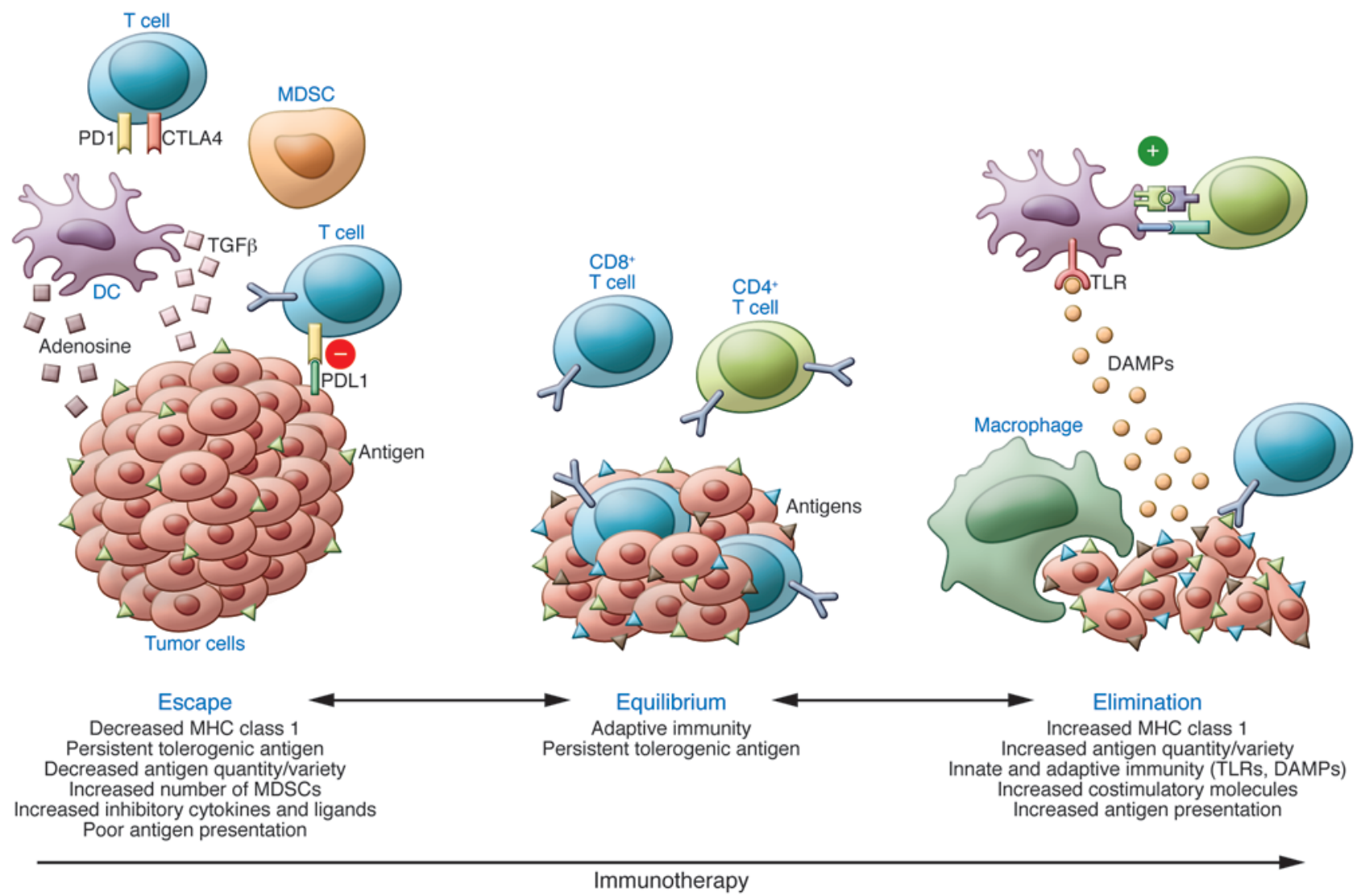

Figure 1

Immunotherapy and the cancer immunoediting hypothesis. In the immunoediting hypothesis, the tumor immune system balance shifts among tumor escape (to mutation-specific antigens), equilibrium, and elimination. Tumor escape (left) occurs in cases with poor antigenic expression, immunosuppressive cytokines, MDSCs, and expression of negative regulatory receptors on T cells (which engage cognate ligands on the tumor). In the equilibrium phase (middle), the tumor and the adaptive immune system coexist. Here, the immune system creates a growth-inhibitory environment and antigenic tumor outgrowths are kept in check. In tumor elimination (right), which often occurs in early tumor development, highly antigenic tumor clones are recognized and eliminated by both innate and adaptive immune systems. Various forms of immunotherapy (e.g., vaccines, adoptive cell transfer, targeted immunotherapeutics) aim to shift the balance from escape and equilibrium to elimination.

directly contributes an effector population of cells. In a study of 93 patients, response rates by RECIST ranged from 49\%-72\%; $22 \%$ had a complete response (11).

By 2006, human peripheral lymphocytes had been engineered with tumor specificity using a TCR, and a study demonstrated efficacy when these lymphocytes were administered to patients with metastatic melanoma (12). A sister approach uses chimeric antigen receptors (CARs), a fusion between the antigen-binding (variable) portion of an antibody and the signaling component of a TCR. Over the past decade, TCR-based adoptive immunotherapy has successfully treated patients with melanoma and synovial cell sarcoma (13). Adoptive immunotherapy using CARs has been effective in neuroblastoma, and more recently, success was reported using CD19-specific CARs in advanced follicular lymphoma, refractory chronic lymphocytic leukemia, and pediatric acute lymphocytic leukemia (14-17). These profound responses occur in a subset of patients; the challenge of ongoing clinical trials using CARs is to increase the number of complete and durable responses. This promising approach is rapidly being developed in a variety of solid tumors, including prostate cancer, non-small cell lung cancer, glioblastoma, and breast and ovarian cancer.
In terms of cancer immunoediting, delivering effector $\mathrm{T}$ cells is akin to adding an immune component capable of elimination. The presence of natural "off" switches in the immune system counteracts the systemic activation of T cells. Peripheral T cells can be induced into anergy or exhaustion, as they would in autoimmunity and chronic viral infection, respectively (18). As a result, T cells may express predominantly negative regulatory receptors when they arrive at distant tumor sites. As T cells repeatedly encounter antigens, they proceed down a differentiation sequence from naive to central and effector memory that correlates with decreased proliferation, cytokine release, and antitumor function (19). Thus, the immune escape state prevails.

Targeted immunotherapeutics. Molecular therapeutics (e.g., monoclonal antibodies) can shift the immune system toward antitumor activity by manipulating immune checkpoints. The most successful therapy thus far has been ipilimumab (Figure 2), a fully human monoclonal antibody that inhibits CTLA4, a negative regulatory molecule that inhibits function of the immune system (20). The rationale is to trigger preexisting antitumor immune responses that are lying dormant.

In a phase 3 study of 676 metastatic melanoma patients that compared the efficacy of ipilimumab, gp100 peptide vaccine, or 
their combination, the median survival of patients receiving ipilimumab alone or in combination with the vaccine was four months longer than patients receiving the vaccine alone (21). A subsequent phase 3 clinical trial combining ipilimumab with standard chemotherapy showed a clear improvement in both overall and progression-free survival compared with standard chemotherapy alone (22). Ipilimumab is currently being studied in cancers of the prostate (in combination with vaccine therapy) (23), cervix, pancreas, lung, ovary, bladder, and breast.

Several other molecules and pathways act as immune switches. Most recently, clinicians have targeted PD-1, another negative regulatory receptor expressed on T cells. This phase 2 trial enrolled patients with 5 different solid tumors and observed $20 \%-25 \%$ objective responses in melanoma, renal cell carcinoma, and nonsmall cell lung cancer (24). It is unknown why a response was observed in lung cancer, but the mechanism may relate to the high number of mutations compared with other epithelial malignancies. In any case, since the treatment was effective in only a minority of patients, these effects may be bolstered by the addition of RT.

Switching a single immune checkpoint may be insufficient to induce an antitumor response and may explain some of the failures of targeting single immune regulatory checkpoints like PD-1 or CTLA4. Tumors already in immune escape are less likely to benefit from activation of a single immune checkpoint, as other factors such as poor antigenicity and additional negative immune regulators predominate. Tumors on the border between equilibrium and elimination states may be more likely to respond.

Another challenge is the maximum tolerated immune activation. Although agents such as ipilimumab alter tumor microenvironments, our choices of immunotherapeutics and adjuvants such as RT are mitigated by concern for "on-target" toxicity concerns. These side effects, which are due to an immune response against appropriate healthy targets, have been reported in immunotherapy (including ipilimumab) (22) and may limit the simultaneous targeting of multiple immune regulatory pathways.

\section{Immunomodulatory properties of RT}

The term abscopal, deriving from the latin $a b$ (away from) and the ancient Greek skopos (target) was introduced in 1953 (25) to describe a rare phenomenon in which the effects of RT are seen outside of the treated area. Anecdotally, tumors outside of RT treatment fields have been said to shrink as a result of a putative systemic inflammatory or immune response provoked by RT (26-28). In contrast, others described RT as an immunosuppressant (29). Lymphocytes are exquisitely sensitive to the effects of RT (30-32), and irradiating the tumor target could potentially eliminate antitumor immune activity. This, along with the general ineffectiveness of RT on local control and of early immunotherapies on systemic disease, dampened enthusiasm for pursuing the combination.

In 2012, two case reports highlighted the immunoadjuvant effect of RT in melanoma, which was classically thought to be an immunogenic tumor $(33,34)$. Following RT, preexisting tumor-specific antibody levels rose, $\mathrm{T}$ cell activation markers were enriched, and new antitumor antibodies were identified. In one case, the patient had a presumed abscopal response after RT alone and a second response after combined RT and targeted immunotherapy. In the second case, the patient progressed slowly despite treatment with targeted immunotherapy, but then exhibited a response after palliative radiotherapy and additional treatment with targeted immunotherapy. These initial anecdotal reports focused on the potential for radiation to spark a systemic antitumor immune response. Augmenting immune activity may also potentiate the local effects of RT. The possibility of improving treatment of both local and widespread disease makes this combination worthy of closer examination.

\section{How RT counters immune evasion}

Antigen quantity, variety, and presentation. What can be done to encourage this reverse transition from escape to equilibrium to elimination? Results from phase 1 and 2 vaccine studies of HLA-restricted multiple TUMAP vaccines in renal cell carcinoma suggest that robustness of antitumor response to vaccines correlates with the number of antigens to which the immune system mounts a response (35). The genomic complexity of malignancies, highlighted by numerous somatic mutations (36), is a source of numerous "private" antigens not otherwise expressed in normal tissue (37). In vitro and in vivo mouse studies indicate that tumor irradiation exposes this complex antigenic environment by generating new peptides and increasing the pool of intracellular peptides for cross-presentation $(38,39)$. It is conceivable that without the generation of these new antigens by RT, the persistent tolerogenic antigens are most expressed, impeding the antitumor immune response.

Tumors also evade antigen presentation. Expression of MHC-I molecules, critical for antigen recognition by cognate $\mathrm{CD} 8^{+} \mathrm{TCR}$, is diminished in tumors. This has been especially well documented in breast, prostate, and lung cancer (40). Tumors can also lose the ability to process antigens intracellularly or can select for less antigenic clones. Radiation, on the other hand, augments MHC-I expression $(38,39,41)$. Although dendritic cells have been shown to naturally infiltrate certain tumors, including melanoma and cancers of the breast, stomach, thyroid, and prostate (42), ablative RT doses can recruit hematopoietic and dendritic cells into tumor (43). However, the recruitment of dendritic cells can actually inhibit the adaptive immune response and promote tumor growth $(44,45)$. Preexisting suppressive dendritic cells in draining lymph nodes can disrupt $\mathrm{T}$ cell proliferation at these sites. Likewise, the tumor microenvironment can suppress dendritic cell activity. For example, constituent transcription factor activation (e.g., STAT3) in tumors leads to cytokine release (e.g., TGF- $\beta$, VEGF, adenosine), inhibiting maturation of APCs and contributing to tumor evasion $(46,47)$.

Bridging innate and adaptive immunity. Immunotherapy often seems to fail due to an inability to transition from equilibrium to elimination phase. Radiation establishes a mechanistic link between an innate and adaptive immune response that may overcome this failure. TLR4 has been shown to play a central role in this link in animal models (Figure 2 and ref. 48). Apetoh et al. demonstrated that RT causes dying tumor cells to release highmobility group box 1 (HMGB-1), a well-described "danger signal" that binds TLR4 (48). Tumor antigen processing and presentation on MHC-I molecules is dependent on the HMGB-1/TLR4 interaction. Mice lacking this pathway could not be successfully vaccinated against tumors, and RT was not effective in treating these tumors. These results are supported by a study combining RT and a different TLR, TLR7, in a mouse model of lymphoma. Compared with either treatment alone, combination therapy improved survival in a $\mathrm{CD}^{+} \mathrm{T}$ cell-dependent manner (49).

The identification of TLR4 as a link between innate and adaptive immunity after radiotherapy was fueled by clinical observations. Node-positive breast cancer patients with inactivating 


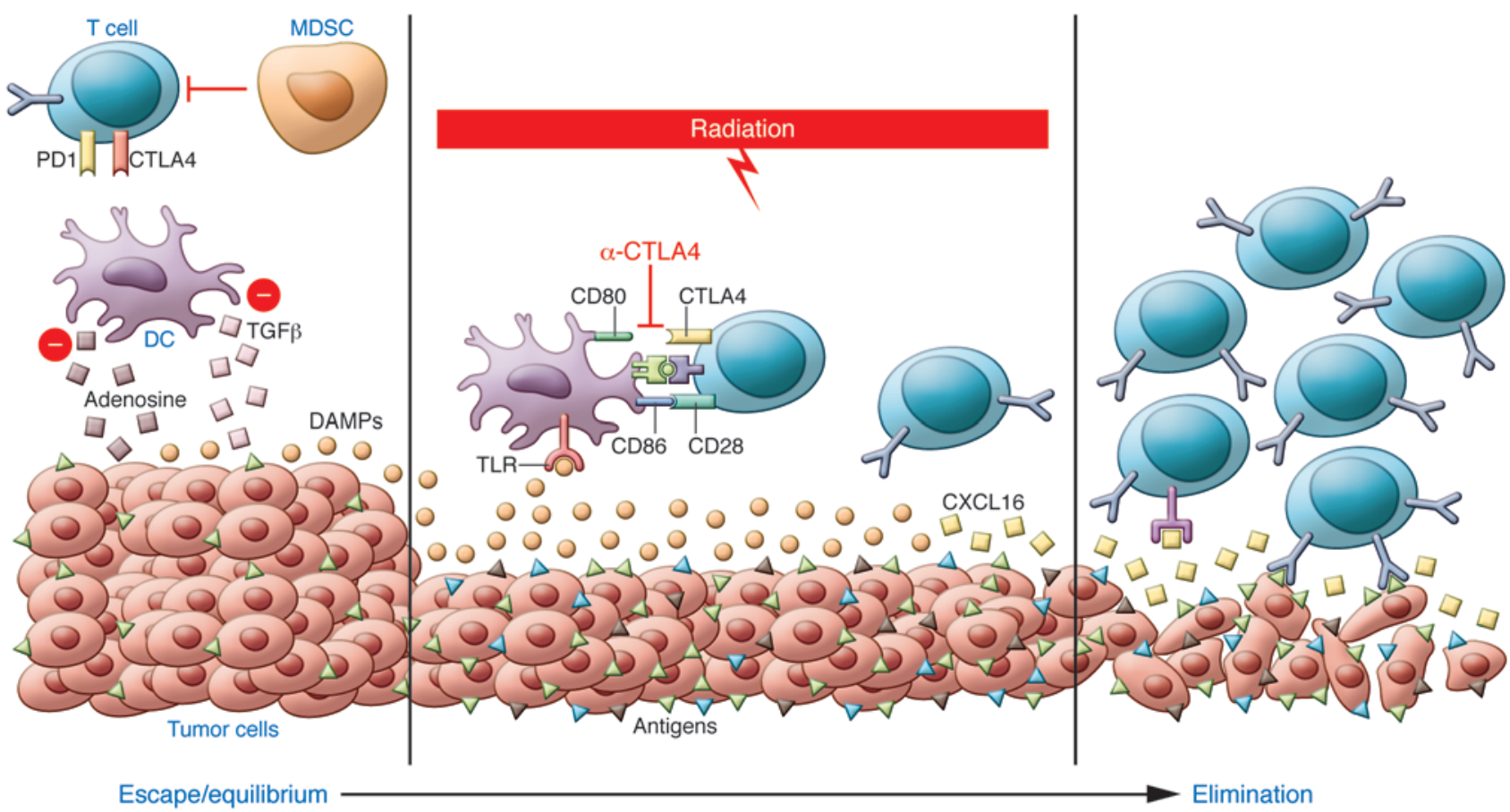

Figure 2

The role of RT in induction of the antitumor immune response. At baseline, both the tumor immune microenvironment and the poor antigenicity of the tumor allow it to escape immune recognition. Targeted RT can induce increased antigenic expression, release pro-inflammatory cytokines (e.g., CXCL16) that recruit immune cells, promote antigen cross-presentation (HMGB-1 via TLR4), and induce tumor expression of death receptors. Anti-CTLA4-targeted immunotherapy can enhance the adaptive immune component by promoting antigen cross-presentation and T cell activation. Used together, RT and immunotherapy may have synergistic effects and may shift the tumor immune system balance toward elimination.

TLR4 mutations have a worse prognosis after chemoradiation than those without the mutation (50). A similar observation was noted in patients with head and neck squamous cell carcinomas who bear these mutations (51). More fundamentally, in a series of esophageal squamous cell carcinoma patients, preoperative chemoradiation increased cancer-specific $\mathrm{T}$ cell responses and serum levels of HMGB-1, the latter of which correlated with overall survival. Again, this suggests a link between innate and adaptive responses (52).

Inducing a T cell response. The most recent and promising immunotherapeutics shift the tumor microenvironment in favor of $\mathrm{T}$ cell activation by blocking negative inhibitory molecules (CTLA4, PD-1, TIM3, LAG3) and stromal cells (myeloid-derived suppressor cells [MDSCs]) (Figure 2 and ref. 53). Cell-based immunotherapies bypass the initial activation step altogether via adoptive transfer of ex vivo activated tumor-specific lymphocytes, which are typically equipped with costimulatory molecules. However, even these lymphocytes can be negatively regulated and nudged toward tolerance once they arrive in the tumor microenvironment. By inducing the release of danger signals such as HMGB-1, radiation can putatively promote activation and maturation of APCs and help shift the balance toward $\mathrm{T}$ cell activation (Figure 2 and ref. 54). In support of this, naive $T$ cells in draining lymph nodes of tumors irradiated with ablative doses proliferate to a far greater degree than in lymph nodes of nonirradiated tumors (55).

The development of a systemic antitumor immune response has been described as concomitant immunity. For example, in a murine model of concomitant immunity wherein mice harbored immuno- genic primary tumors, tumor challenge at a distant site was rejected, apparently via mediation by effector $\mathrm{CD}^{+} \mathrm{T}$ cells and regulatory $\mathrm{CD} 4^{+} \mathrm{CD} 25^{+} \mathrm{T}$ cells (2). RT, likewise, may induce concomitant immunity where it previously did not exist (e.g., RT-induced in situ vaccination). In an animal model of breast cancer, ablative RT of a primary tumor prevented the growth of metastatic tumor colonies in the lung, an effect that was dependent on the presence of $\mathrm{CD}^{+} \mathrm{T}$ cells (55). Other animal models have displayed augmentation of systemic antitumor immunity following local RT, with reduction, control, or elimination of distant metastases, especially in combination with immunotherapy (55-63). As a result, the combination of RT and immunotherapy in locally advanced disease is now being studied in clinical trials (Table 1).

In addition to promoting tumor recognition by preexisting tumor-specific T cells, RT can also generate tumor-specific cytotoxic $\mathrm{T}$ cells (64). Cytotoxic $\mathrm{T}$ cells are more easily recruited to the irradiated tumor site due to the release of chemokines such as CXCL16, as demonstrated in breast cancer cells (ref. 65 and Figure 2). Irradiated tumors upregulate death receptors (e.g., FAS), promoting the cytotoxic effect of T cells at the tumor site (66). The immune system, unleashed of its negative regulatory pathways at the site of the irradiated tumor, is a powerful antitumor agent locally. These studies suggest a role for immunomodulatory agents in promoting improved RT efficacy.

In fact, an intact immune system may be critical for RT to exert its maximal antitumor effect. In a mouse model of melanoma, highdose, single-fraction RT slowed the growth of small tumors in immunocompetent mice, but not in nude mice. Investigators demon- 


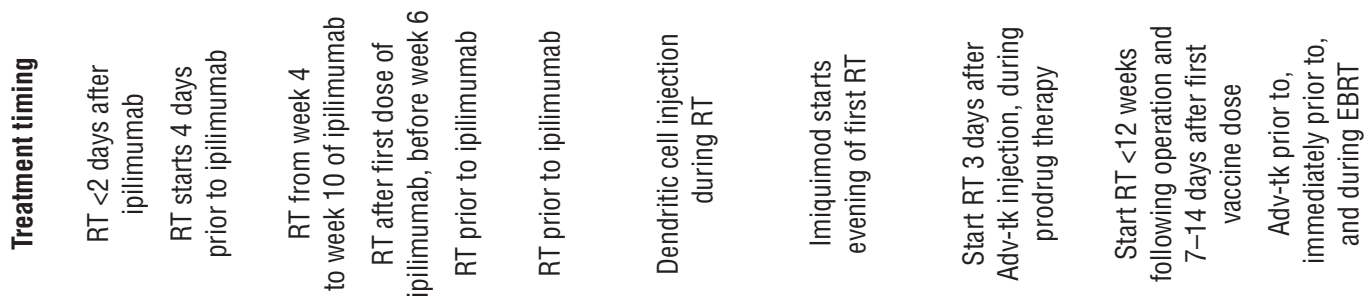

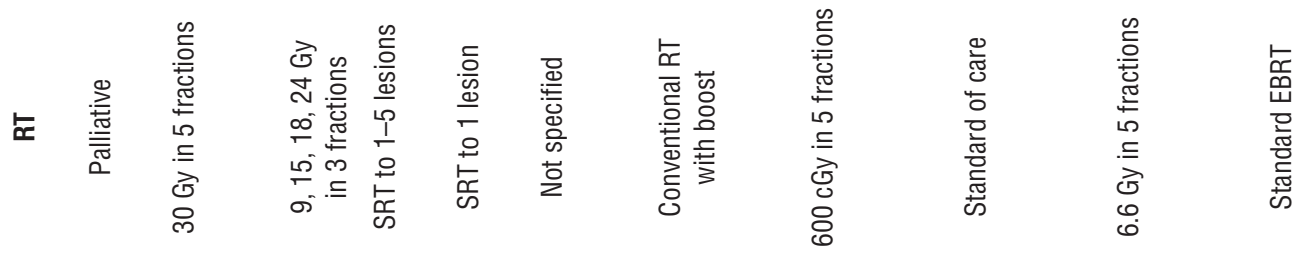

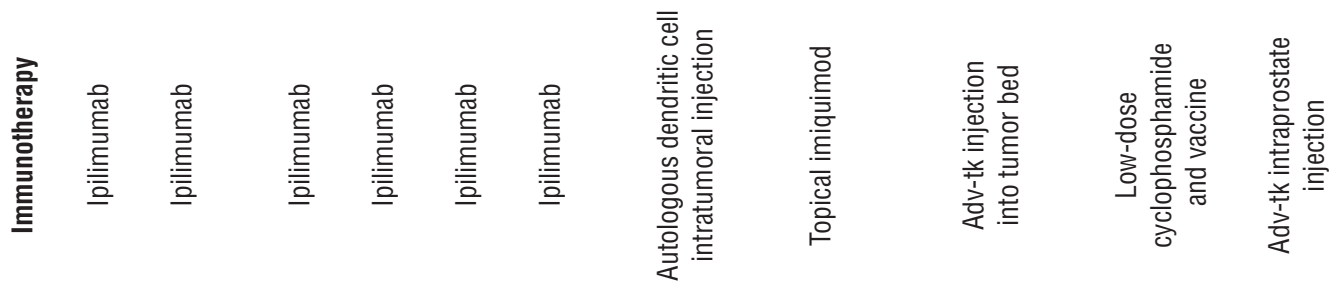

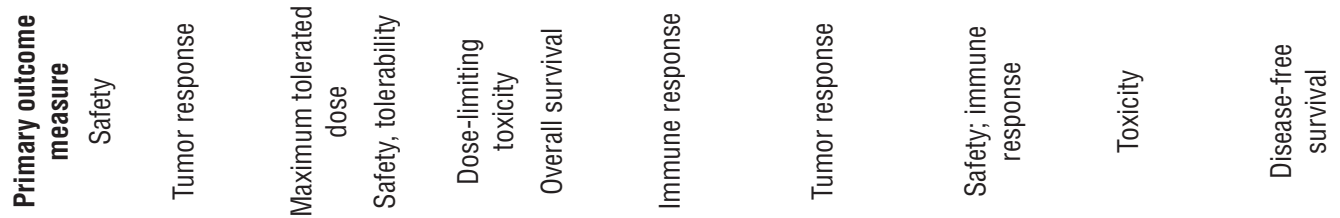

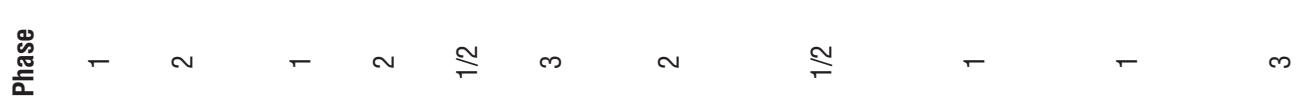

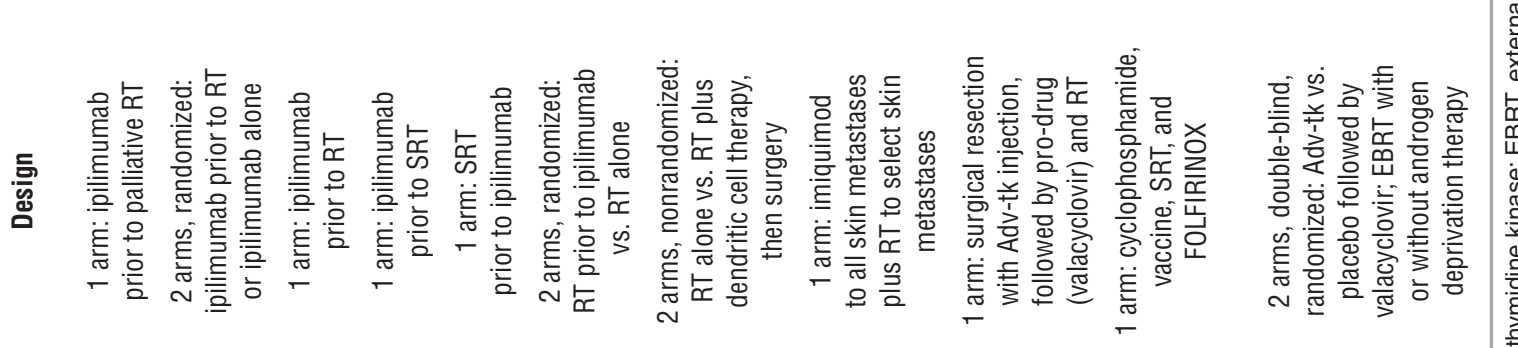

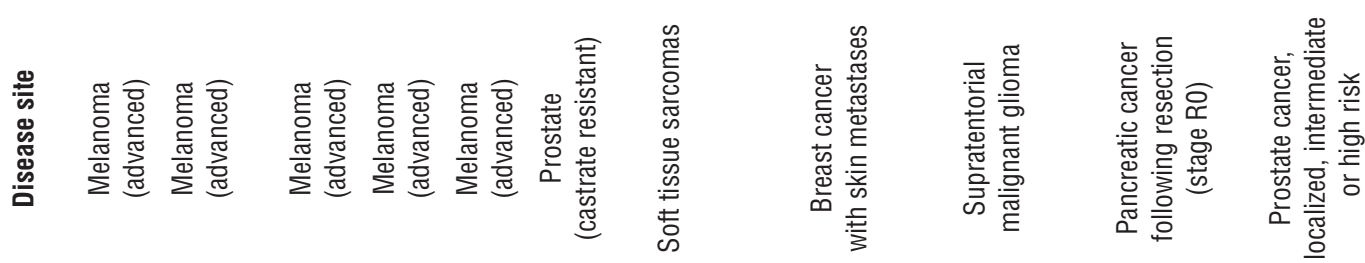

总

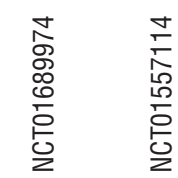

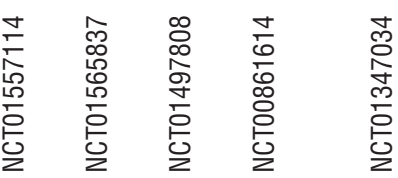

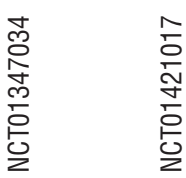

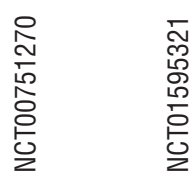

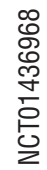


strated that $\mathrm{CD}^{+} \mathrm{T}$ cells were necessary for this slowed growth and improved survival of immunocompetent mice after RT $(55,64)$. A more recent study in a mouse model of intracranial glioma demonstrated that combining RT and anti-PD-1 antibody prolonged survival - an effect that was not seen with either modality alone and that was abrogated by depletion of $\mathrm{CD}^{+}$or $\mathrm{CD}^{+} \mathrm{T}$ cells (41).

\section{Clinical trials of combination RT and immunotherapy}

The enthusiasm for the combined approach of RT and immunotherapy has led to clinical trials (Table 1). In particular, the FDA approval of ipilimumab for use in metastatic melanoma has led to the design of phase 1 and 2 trials that combined ipilimumab treatment with RT, most of which will primarily assess safety and early efficacy. The most informative of the studies combining RT and ipilimumab may be a two-arm study examining the benefit of adding RT to ipilimumab (ClinicalTrials.gov identifier: NCT01689974). It will be difficult to assess the contribution of RT to systemic immune response or efficacy in single-arm combination studies.

Other current trials are exploring dendritic cell vaccination (both intratumoral and systemic) in combination with RT, and 1 trial is studying the use of topical immunotherapy together with RT in breast cancer skin metastases. RT in all trials is delivered using conventionally fractionated regimens or stereotactic techniques. For adoptive cell transfer therapy, local immunoadjuvant RT techniques (not including whole body irradiation as an immunodepleting regimen) have not yet been studied in humans but are ripe for research.

The combination of RT and immunotherapy has proven to be safe in a controlled setting. In a phase 1 study of 12 patients, investigators demonstrated the safety of combining stereotactic body RT and interleukin-2, an FDA-approved immunotherapy for metastatic renal cell carcinoma and melanoma (67). Furthermore, response rates compared favorably with historical data on IL-2 alone, and responding patients exhibited characteristic T cell populations in peripheral blood prior to treatment. A similar single-arm phase $1 / 2$ study also demonstrated safety and feasibility of combining local RT with a TLR9 agonist (68).

As phase 2 studies report early results, a critical appraisal of clinical response rates is needed. Current imaging modalities may be unable to distinguish between inflammation induced by RT or immunotherapy and persistent disease. Early reports will not include survival data, but immune correlative studies that include peripheral blood examination for cytokine levels, antigen-specific $\mathrm{T}$ cell responses, and phenotypes of immune cells, as well as pathologic evidence of antitumor immunity, will be essential. Many of the studies listed in Table 1 plan to examine peripheral markers of immune activation.

To understand the pathologic changes in the tumor microenvironment, studies of RT and immunotherapy should be considered in the preoperative setting. One such investigation is underway for soft tissue sarcomas (ClinicalTrials.gov identifier: NCT01347034). In this two-arm study, conventionally fractionated RT is followed by autologous dendritic cells administered prior to surgical resection. However, in accordance with standards of care, the experimental therapy in this study is dendritic cells rather than RT. In addition to soft tissue sarcomas, other sites prime for clinicopathologic studies, given the routine use of preoperative RT, are rectal and esophageal cancers as well as head and neck and lung malignancies that require salvage surgery after chemoradiation.
While the majority of the studies listed in Table 1 are designed for the metastatic setting, there may be a unique therapeutic window in locally advanced disease. In this setting, preoperative or definitive RT in combination with immunotherapy may induce in situ vaccination against micrometastatic disease as a clinical model for concomitant immunity. In locally advanced tumors that have a high propensity for metastasis, such an approach may improve distant metastasis-free survival.

One challenge is finding a place for the combination of RT and immunotherapy in the context of current standards of care. For instance, one study in pancreatic cancer will examine the safety of a cancer vaccine administered prior to adjuvant RT and chemotherapy (ClinicalTrials.gov identifier: NCT01595321). In this study, patients have had complete resections of their tumors, so RT cannot induce in situ vaccination. Furthermore, aggressive adjuvant chemotherapy may eliminate any antitumor immune response.

\section{Combining RT and immunotherapy: logistical challenges}

Timing of RT with respect to immunotherapy. The timing of RT is a function of the expected immune response at the tumor site. Because of its cytotoxic nature, administering to the tumor target could disrupt an ongoing antitumor cellular immune response. Conversely, having an active immunoadjuvant within the tumor microenvironment could maximize the effects of RT. Targeted agents such as ipilimumab could be administered prior to RT to potentiate a radiation-induced immune response; however, if ipilimumab alone is administered well before RT and has already incited an immune response at the tumor site, the cytotoxic effect of RT on lymphocytes may be detrimental. Furthermore, targeting immune checkpoints of T cell activation may be less effective if RT has not already generated de novo antigens and broken preexisting peripheral tolerance. In the case of adoptively transferred $\mathrm{T}$ cell immunotherapy, RT should presumably be administered prior to $\mathrm{T}$ cell transfer to avoid disruption of the $T$ cell response at the tumor site.

The timing of vaccine therapy is similarly under question. Vaccine therapy is administered either systemically or intratumorally. In the latter scenario, the immune-stimulatory effects of coincident radiation may stimulate the antivaccine inflammatory response necessary for antigen presentation. Conversely, if delivered well after vaccine therapy, RT may disrupt the development of an antivaccine cellular immune response. In ongoing trials, vaccine therapy is administered either a few days prior to RT, during RT, or both. Although this has not been well studied, delivering RT well before vaccine therapy may be futile, as the vaccine may not benefit from potential transient immune-activating qualities of RT.

Fractionation of RT regimens. When priming the immune system, is it better to use conventional fractionation or hypofractionated regimens? Historically, RT has been administered in daily fractions of approximately 1.5-2.0 Gy over a period of weeks. The relatively small daily dose is designed to allow for normal tissue repair. Techniques such as intensity-modulated RT have permitted more conformal dose delivery, which more tightly matches radiation dose clouds to the shape of the tumor target and minimizes normal tissue exposure. Consequently, there has been a trend toward hypofractionation, which involves the delivery of larger daily doses in fewer fractions to achieve a high, biologically effective dose. Currently, stereotactic radiotherapy (SRT), defined as RT delivered in 5 or less fractions, is used for tumors in the brain, lung, liver, bone, and more. 
Preclinical comparison studies of different fractionation regimens in combination with immunotherapy have been equivocal (69). In a mouse model of melanoma, a single dose of radiation in combination with $\mathrm{T}$ cell immunotherapy was more effective than a fractionated scheme (55). Conversely, in combination with TLR7 agonism, a regimen of five fractions of $2 \mathrm{~Gy}$ each completely eradicated tumors in a mouse model of lymphoma, while a single 10-Gy fraction only slowed growth (49). It is not clear whether the different fractionated schemes actually deliver the same biologically equivalent dose to the tumor (e.g., $20 \mathrm{~Gy}$ ' 1 does not equal 5 Gy' 4). Furthermore, it is unclear how these fractionation schemes in animal models parallel conventional or hypofractionated RT in humans.

Total radiation dose. Total radiation dose is also a logistical hurdle in combining RT and immunotherapy. The goal is to provide sufficient radiation to trigger an effective antiinflammatory response and, in turn, a tumor-specific immune response. For instance, in an IFN- $\gamma$-deficient mouse model, production of IFN- $\gamma$ after RT is important in switching the immune system into an antitumor position (70). IFN- $\gamma$ production led to expression of molecules important for $\mathrm{T}$ cell homing and antigen presentation. Escalating radiation doses increased the level of IFN- $\gamma$ production, although at the highest doses there was concern about a simultaneous rise in regulatory T cells (69). These early studies suggest a benefit of higher radiation doses, with the caveat of potential development of regulatory immune pathways and cells.

Choosing a site for RT. The degree of immune response to RT may also depend on the site of irradiation. For example, the activity of the immune system (especially dendritic cells) in the skin, gut, and lung is unique because of the exposure of these organs to external pathogens (71). Immune phenomena in the liver are different due to its chronic and persistent exposure to toxic metabolites as well as antigens from the gastrointestinal tract. The testis is an immune-privileged organ (72), and our understanding of immune phenomena in the central nervous system is evolving (73). RT may result in distinct immune phenomenon at each site. Identifying which irradiated sites best elicit a local and systemic tumor response will be critical in identifying patient groups that could maximally benefit from combined RT and immunotherapy.

\section{Conclusions}

The ability of RT to invoke a systemic immune response and the immune system's role in the local effects of RT underscore the potential clinical utility of this combination approach. As ongoing trials begin to report safety and efficacy data, it will be critical to dissect this information to determine the most appropriate logistical combinations of immunotherapy and RT and to identify the patient subgroups that may benefit most from this approach.

\section{Acknowledgments}

The authors thank Mary Leonard for contributions to figure illustration.

Address correspondence to: Anusha Kalbasi, Hospital of the University of Pennsylvania, PCAM-4 West, 3400 Civic Center Blvd., Philadelphia, Pennsylvania 19104, USA. Phone: 215.662.2428; Fax: 215.615.1658; E-mail: Anusha.Kalbasi@uphs.upenn.edu.
1. Rosenberg SA, Robbins P, Restifo NP. Cancer immunotherapy. In: Rosenberg SA, Lawrence TS, Devita VT, eds. DeVita, Hellman, and Rosenberg's Cancer: Principles and Practice of Oncology. 9th ed. 2011:333-344.

2. Turk MJ, Guevara-Patiño JA, Rizzuto GA, Engelhorn ME, Sakaguchi S, Houghton AN. Concomitant tumor immunity to a poorly immunogenic melanoma is prevented by regulatory T cells. J Exp Med. 2004;200(6):771-782.

3. Berendt M, North R. T-cell-mediated suppression of anti-tumor immunity. An explanation for progressive growth of an immunogenic tumor. J Exp Med. 1980;151(1):69-80.

4. Dunn GP, Bruce AT, Ikeda H, Old LJ, Schreiber RD. Cancer immunoediting: from immunosurveillance to tumor escape. Nat Immunol. 2002;3(11):991-998.

5. Schreiber RD, Old LJ, Smyth MJ. Cancer immunoediting: integrating immunity's roles in cancer suppression and promotion. Science. 2011;331(6024):1565-1570

6. Small EJ, et al. Placebo-controlled phase III trial of immunologic therapy with sipuleucel-T (APC8015) in patients with metastatic, asymptomatic hormone refractory prostate cancer. J Clin Oncol. 2006;24(19):3089-3094.

7. Kantoff PW, et al. Overall survival analysis of a phase II randomized controlled trial of a Poxviral-based PSA-targeted immunotherapy in metastatic castration-resistant prostate cancer. J Clin Oncol. 2010;28(7):1099-1105.

8. Walter $S$, et al. Multipeptide immune response to cancer vaccine IMA901 after single-dose cyclophosphamide associates with longer patient survival [published online ahead of print July 29, 2012]. Nat Med. doi:10.1038/nm.2883.

9. Hailemichael Y, et al. Persistent antigen at vaccination sites induces tumor-specific CD8+ T cell sequestration, dysfunction and deletion. Nat Med. 2013;19(4):465-472.
10. Rosenberg SA, et al. Use of tumor-infiltrating lymphocytes and interleukin- 2 in the immunotherapy of patients with metastatic melanoma. A preliminary report. NEnglJ Med. 1988;319(25):1676-1680.

11. Dudley ME, et al. Adoptive cell therapy for patients with metastatic melanoma: evaluation of intensive myeloablative chemoradiation preparative regimens. J Clin Oncol. 2008;26(32):5233-5239.

12. Morgan RA, et al. Cancer regression in patients after transfer of genetically engineered lymphocytes. Science. 2006;314(5796):126-129.

13. Robbins PF, et al. Tumor regression in patients with metastatic synovial cell sarcoma and melanoma using genetically engineered lymphocytes reactive with NY-ESO-1. J Clin Oncol. 2011;29(7):917-924.

14. Pule MA, et al. Virus-specific T cells engineered to coexpress tumor-specific receptors: persistence and antitumor activity in individuals with neuroblastoma. Nat Med. 2008;14(11):1264-1270.

15. Kochenderfer JN, et al. Eradication of B-lineage cells and regression of lymphoma in a patient treated with autologous $T$ cells genetically engineered to recognize CD19. Blood. 2010; 116(20):4099-4102

16. Porter DL, Levine BL, Kalos M, Bagg A, June $\mathrm{CH}$. Chimeric antigen receptor-modified $\mathrm{T}$ cells in chronic lymphoid leukemia. $N$ Engl J Med. 2011;365(8):725-733

17. Grupp S, et al. Chimeric antigen receptor-modified $\mathrm{T}$ cells for acute lymphoid leukemia. $N$ Engl J Med. 2013;368(16):1509-1518.

18. Baitsch L, Fuertes-Marraco S a, Legat A, Meyer C, Speiser DE. The three main stumbling blocks for anticancer T cells. Trends Immunol. 2012;33(7):364-372.

19. Restifo NP, Dudley ME, Rosenberg SA. Adoptive immunotherapy for cancer: harnessing the T cell response. Nat Rev Immunol. 2012;12(4):269-281.

20. Frauwirth KA, Thompson CB. Activation and inhibition of lymphocytes by costimulation. J Clin Invest. 2002;109(3):295-299.

21. Hodi FS, et al. Improved survival with ipilimumab in patients with metastatic melanoma. $N$ Engl $J$ Med. 2010;363(8):711-723.

22. Robert C, et al. Ipilimumab plus dacarbazine for previously untreated metastatic melanoma. NEngl JMed. 2011;364(26):2517-2526.

23. Madan RA, et al. Ipilimumab and a poxviral vaccine targeting prostate-specific antigen in metastatic castration-resistant prostate cancer: a phase 1 dose-escalation trial. Lancet Oncol. 2012;13(5):501-508.

24. Topalian SL, et al. Safety, activity, and immune correlates of anti-PD-1 antibody in cancer. $N$ EnglJ Med. 2012;366(26):2443-2454.

25. Mole RH. Whole body irradiation; radiobiology or medicine? Br J Radiol. 1953;26(305):234-241.

26. Ehlers G, Fridman M. Case reports: Abscopal effect of radiation in papillary adenocarcinoma. $\mathrm{BrJ}$ Radiol. 1973;46(543):220-222.

27. Ohba K, et al. Abscopal regression of hepatocellular carcinoma after radiotherapy for bone metastasis. Gut. 1998;43(4):575-577.

28. Wersäll PJ, Blomgren H, Pisa P, Lax I, Kälkner KM, Svedman C. Regression of non-irradiated metastases after extracranial stereotactic radiotherapy in metastatic renal cell carcinoma. Acta Oncol. 2006;45(4):493-497.

29. Order SE. The effects of therapeutic irradiation on lymphocytes and immunity. Cancer. 1977; 39(2 suppl):737-743.

30. Chee C, Ilbery P, Rickinson A. Depression of lymphocyte replicating ability in radiotherapy patients. BrJ Radiol. 1974;47(553):37-43.

31. Wasserman J, Blomgren $H$. Effect of radiation therapy and in vitro $x$-ray exposure on lymphocyte subpopulations and their functions. Am J Clin Oncol. 1982;5(2):195-208.

32. Wasserman J, Blomgren H, Rotstein S, Petrini B, Hammarström S. Immunosuppression in irradiated breast cancer patients: in vitro effect of cyclooxygenase inhibitors. Bull N Y Acad Med. 1989;65(1):36-44.

33. Postow MA, et al. Immunologic correlates of the 
abscopal effect in a patient with melanoma. NEngl JMed. 2012;366(10):925-931.

34. Stamell EF, Wolchok JD, Gnjatic S, Lee NY, Brownell I. The abscopal effect associated with a systemic anti-melanoma immune response. Int $J$ Radiat Oncol Biol Phys. 2013;85(2):293-295.

35. Walter $\mathrm{S}$, et al. Multipeptide immune response to cancer vaccine IMA901 after single-dose cyclophosphamide associates with longer patient survival [published online ahead of print July 29, 2012]. Nat Med. doi:10.1038/nm.2883.

36. Sjöblom T, et al. The consensus coding sequences of human breast and colorectal cancers. Science. 2006;314(5797):268-274.

37. Segal NH, et al. Epitope landscape in breast and colorectal cancer. Cancer Res. 2008;68(3):889-892.

38. Reits EA, et al. Radiation modulates the peptide repertoire, enhances MHC class I expression, and induces successful antitumor immunotherapy. JExp Med. 2006;203(5):1259-1271.

39. Sharma A, et al. $\gamma$-Radiation promotes immunological recognition of cancer cells through increased expression of cancer-testis antigens in vitro and in vivo. PLoS One. 2011;6(11):e28217.

40. Marincola F, Jaffee E, Hicklin D, Ferrone S. Escape of human solid tumors from T-cell recognition: molecular mechanisms and functional significance. Adv Immunol. 2000;74:181-273.

41. Zeng J et al. Anti-PD-1 blockade and stereotactic radiation produce long-term survival in mice with intracranial gliomas. Int J Radiat Oncol Biol Phys. 2013;pii:S0360-3016(13)00004-7.

42. Vicari AP, Caux C, Trinchieri G. Tumour escape from immune surveillance through dendritic cell inactivation. Semin Cancer Biol. 2002;12(1):33-42.

43. Burnette BC, et al. The efficacy of radiotherapy relies upon induction of type i interferon-dependent innate and adaptive immunity. Cancer Res. 2011;71(7):2488-2496.

44. Norian LA, et al. Tumor-infiltrating regulatory dendritic cells inhibit CD8 $+\mathrm{T}$ cell function via L-arginine metabolism. Cancer Res. 2009;69(7):3086-3094.

45. Aspord C, et al. Breast cancer instructs dendritic cells to prime interleukin 13 -secreting $\mathrm{CD}^{+} \mathrm{T}$ cells that facilitate tumor development. J Exp Med 2007;204(5):1037-1047.

46. Pardoll D. Does the immune system see tumors as foreign or self? Annu Rev Immunol. 2003;21:807-839.

47. Mellman I, Coukos G, Dranoff G. Cancer immunotherapy comes of age. Nature. 2011; 480(7378):480-489.
48. Apetoh L, et al. Toll-like receptor 4-dependent contribution of the immune system to anticancer chemotherapy and radiotherapy. Nat Med. 2007;13(9):1050-1059.

49. Dovedi SJ, et al. Systemic delivery of a TLR7 agonist in combination with radiation primes durable anti-tumor immune responses in mouse models of lymphoma. Blood. 2013;121(2):251-259.

50. Ghiringhelli F, et al. The TLR4 polymorphism predicts the response of breast cancer patients to anthracyclins. J Clin Oncol. 2007;25(18S):10612.

51. Bergmann C, et al. Toll-like receptor 4 single-nucleotide polymorphisms Asp299Gly and Thr399Ile in head and neck squamous cell carcinomas. J Trans Med. 2011;9:139.

52. Suzuki Y, et al. Immunogenic tumor cell death induced by chemoradiotherapy in patients with esophageal squamous cell carcinoma. Cancer Res. 2012;72(16):3967-3976.

53. Whiteside TL. Immune responses to malignancies. J Allergy Clin Immunol. 2010;125(2 suppl 2):S272-S283.

54. Messmer D, et al. High mobility group box protein 1: an endogenous signal for dendritic cell maturation and Th1 polarization. J Immunol. 2004;173(1):307-313.

55. Lee Y, et al. Therapeutic effects of ablative radiation on local tumor require $\mathrm{CD} 8+\mathrm{T}$ cells: changing strategies for cancer treatment. Blood. 2009;114(3):589-595.

56. Camphausen $\mathrm{K}$, et al. Radiation abscopal antitumor effect is mediated through p53. Cancer Res. 2003;63(8):1990-1993

57. Blanquicett C, et al. Antitumor efficacy of capecitabine and celecoxib in irradiated and lead-shielded, contralateral human $\mathrm{BxPC}-3$ pancreatic cancer xenografts: clinical implications of abscopal effects. Clin Cancer Res. 2005;11(24 pt 1):8773-8781.

58. Demaria $\mathrm{S}$, et al. Ionizing radiation inhibition of distant untreated tumors (abscopal effect) is immune mediated. Int J Radiat Oncol Biol Phys. 2004;58(3):862-870.

59. Dewan MZ, et al. Fractionated but not single-dose radiotherapy induces an immune-mediated abscopal effect when combined with anti-CTLA-4 antibody. Clin Cancer Res. 2009;15(17):5379-5388.

60. Vlachaki MT, et al. Enhanced therapeutic effect of multiple injections of HSV-TK + GCV gene therapy in combination with ionizing radiation in a mouse mammary tumor model. Int J Radiat Oncol Biol Phys. 2001;51(4):1008-1017.

61. Liu LL, Smith MJ, Sun BS, Wang GJ, Redmond HP, Wang JH. Combined IFN-gamma-endostatin gene therapy and radiotherapy attenuates primary breast tumor growth and lung metastases via enhanced CTL and NK cell activation and attenuated tumor angiogenesis in a murine model. Ann Surg Oncol. 2009;16(5):1403-1411.

62. Pilones KA, Kawashima N, Yang AM, Babb JS, Formenti SC, Demaria S. Invariant natural killer $\mathrm{T}$ cells regulate breast cancer response to radiation and CTLA-4 blockade. Clin Cancer Res. 2009;15(2):597-606.

63. Demaria $S$, et al. Immune-mediated inhibition of metastases after treatment with local radiation and CTLA-4 blockade in a mouse model of breast cancer. Clin Cancer Res. 2005;11(2 pt 1):728-734.

64. Takeshima $\mathrm{T}$, et al. Local radiation therapy inhibits tumor growth through the generation of tumor-specific CTL: its potentiation by combination with Th1 cell therapy. Cancer Res. 2010; 70(7):2697-2706

65. Matsumura S, et al. Radiation-induced CXCL16 release by breast cancer cells attracts effector $\mathrm{T}$ cells. J Immunol. 2008;180(5):3099-3107.

66. Chakraborty M, Abrams SI, Coleman CN, Camphausen K, Schlom J, Hodge JW. External beam radiation of tumors alters phenotype of tumor cells to render them susceptible to vaccine-mediated T-cell killing. Cancer Res. 2004;64(12):4328-4337.

67. Seung SK, et al. Phase 1 study of stereotactic body radiotherapy and interleukin-2 - tumor and immunological responses. Sci Transl Med. 2012;4(137):137ra74.

68. Kim YH, et al. In situ vaccination against mycosis fungoides by intratumoral injection of a TLR9 agonist combined with radiation: a phase $1 / 2$ study. Blood. 2012;119(2):355-363.

69. Schaue D, Ratikan JA, Iwamoto KS, McBride WH. Maximizing tumor immunity with fractionated radiation. Int J Radiat Oncol Biol Phys. 2012;83(4):1306-1310.

70. Lugade AA, Sorensen EW, Gerber SA, Moran JP, Frelinger JG, Lord EM. Radiation-induced IFNgamma production within the tumor microenvironment influences antitumor immunity. J Immunol. 2008;180(5):3132-3139.

71. Novak N, Bieber T. Dendritic cells as regulators of immunity and tolerance. J Allergy Clin Immunol. 2008;121(2 suppl):S370-S374.

72. Fijak M, Meinhardt A. The testis in immune privilege. Immunol Rev. 2006;213:66-81.

73. Carson MJ, Doose JM, Melchior B, Schmid CD, Ploix CC. CNS immune privilege: hiding in plain sight. Immunol Rev. 2006;213:48-65. 